\title{
Structural Changes in the Urinary Bladder of Streptozotocin-Induced Diabetic Rats and the Possible Protective Role of Insulin
}

Amir M. Bassam Elnagar Ph.D. ${ }^{1,2}$, Suhaidah Ibrahim Ph.D. ${ }^{3}$, Mostafa A. M. Abouelnaga Ph.D. ${ }^{4}$, Amro

Mohamed Soliman M.Sc. ${ }^{5}$

${ }^{1}$ Department of Histology and Cell Biology, Faculty of Medicine, Al-Azhar University, Egypt.

${ }^{2}$ Department of Pathology, Kolej Universiti Insaniah, Kuala Ketil Kedah, Malaysia

${ }^{3}$ Kulliyyah of Medicine and Health Sciences, Kolej Universiti Insaniah, Kuala Ketil, Kedah, Malaysia.

${ }^{4}$ Department of Anatomy, Rabigh Faculty of Medicine, King Abdulaziz University, Saudi Arabia.

${ }^{5}$ Department of Anatomy, Universiti Kebangsaan Malaysia Medical Center, Jalan Yaakob Latif,

Bandar Tun Razak, Kuala Lumpur, Malaysia

\section{ABSTRACT}

Introduction: Diabetes mellitus possesses severe adverse effects on the urinary bladder. Urinary bladder dysfunction is a common health problem affecting diabetic patients causing recurrent infections and urinary incontinence. Objective: To evaluate the histopathological changes in the tissue of urinary bladder in Streptozotocin (STZ) diabetic rats and the protective role of insulin. Methods: Thirty rats were classified into three groups: a control group which received no treatment (Group A), STZ diabetic group (Group B) and Insulin diabetic group (Group C). Animals were sacrificed after six weeks and urinary bladders were harvested and processed for light and electron microscopy. Results: Several histopathological changes were observed in the urinary bladder of the diabetic group including an increase in the thickness of the urothelium, epithelial cells with dark nuclei and large lenticular vesicles, and wide intercellular spaces with numerous collagen fibers. Treatment with insulin reduced the pathological changes induced by STZ. Conclusion: Diabetes mellitus caused significant pathological changes in the urinary bladder of experimental rats. For instance, treating diabetic animals with insulin prevented the development of damaging effects of diabetes on the urinary bladder.

KEYWORDS: Urinary bladder, Diabetes Mellitus, Histology, Insulin

\section{INTRODUCTION}

Diabetes mellitus is a complex of metabolic disorder which is commonly associated with insufficiency of insulin secretion or insulin resistance. It is manifested by hyperglycemia, altered metabolism of lipids, carbohydrates and proteins with an increased risk of complications of vascular diseases. Diabetes mellitus can be classified into two types;

Corresponding author:

Dr. Amro Mohamed Soliman,

Department of Anatomy,

Universiti Kebangsaan Malaysia Medical Center, Jalan Yaakob Latif, Bandar Tun Razak,

56000 Kuala Lumpur, Malaysia.

Tel: (+6)011-12306811

E-mail: dramrsoliman@yahoo.com insulin dependent (IDDM) and non-insulin dependent (NIDDM). The terms IDDM and NIDDM are also known as type I and type 2 diabetes mellitus, respectively. Type I diabetic is mainly caused by Bcell destruction, which is usually immune-mediated. Majority of the IDDM patients develop absolute insulin deficiency and they are liable to develop diabetic ketoacidosis. On the other hand, NIDDM is mainly caused by insulin resistance. NIDDM is so far the most prevalent form of the disease. ${ }^{1-2}$

Urinary bladder dysfunction is one of the major complications of diabetes mellitus. Diabetes induces time-dependent pathological changes in the bladder and its main mechanism is attributed to neurological dysfunction. ${ }^{3}$ Furthermore, evidence of post-voiding residuals is encountered in the bladder of diabetic 
patients which may lead to urinary tract infection. ${ }^{4}$ The common bladder dysfunction is usually due to hyporeflexia of detrusor muscle. However, some patients may have detrusor overactivity without evidence of hyporeflexia. ${ }^{5}$ This overactivity was thought to be due to an increase in beta-nerve growth factor, a gene product responsible for neuronal survival and growth, which leads to alterations in the properties of sodium and potassium channels in the bladder afferent fibers. ${ }^{6}$

A rise in sympathetic markers and tyrosine hydroxylase in addition to a decrease in parasympathetic markers and vesicular acetylcholine transporter were observed in the smooth muscle layer of the bladder in diabetic patients. ${ }^{7}$ This observation was inconsistent with the increased capacity, decreased tone, and dilated state observed in diabetic patients. STZ induced diabetes was reported to have severe adverse effects on the urinary system of experimental animals in previous studies. For instance, STZ induced-diabetes enhanced the endothelialmyofibroblast transition and contributed to the early development and progression of diabetic renal interstitial fibrosis. ${ }^{8}$ Moreover, Diabetes mellitus markedly altered the expression of the gap junction protein $\mathrm{C} \times 43$ urinary bladder. These changes probably contributed to the early development of higher detrusor activity in diabetic rats. ${ }^{9}$ The aim of the present study was to assess the underlying histological changes in experimentally STZ-induced diabetic rats. Moreover, the protective effect of insulin on the bladder of diabetic rats was evaluated.

\section{MATERIALS AND METHODS}

1. Drugs: Streptozotocin (STZ) (Sigma Chemical Co., USA) was used to induce diabetes in animals. Induction of diabetes was confirmed 24 hours after STZ injection by assessment of serum glucose concentration from tail vein blood sample using a standard glucometer (LifeScan, USA). Rats were considered to be diabetic if their blood glucose level was $200 \mathrm{mg} / \mathrm{dl}$ or more according to Rizk et al. ${ }^{5}$ Rats were injected with a $1 \mathrm{U}$ dose of insulin subcutaneously daily. ${ }^{10}$ The insulin used in the experiment was Humulin N, NPH/type (Eli Lilly Co., USA).

2. Animals: The study was conducted in the Animal Lab, Cairo University. Thirty adult male albino rats weighing 200-250 g were obtained from Animal House, Faculty of Medicine, Cairo University. The animals were kept in metabolic cages with rat feed and normal water ad libitum.

3. Experimental design: Animals were divided into three groups: Group (A) a control group which received no treatment, Group (B) a diabetic group which was intraperitoneally injected with $60 \mathrm{mg} / \mathrm{kg}$ body weight of STZ and Group (C) a diabetic group which was treated with insulin. All animals were sacrificed after six weeks of treatment and urinary bladders were harvested. Urinary bladder specimens were fixed in buffered formol saline for preparation of paraffin blocks, $5 \mu \mathrm{m}$ sections were stained by H\&E and Mallory's trichrome stain. ${ }^{11}$ Specimens for electron microscopy were immediately fixed in $2.5 \%$ glutaraldehyde buffered with $0.1 \mathrm{M}$ phosphate buffer at $\mathrm{pH} 7.4$ for 2 hours at $40 \mathrm{C}$. Then, specimens were embedded in Embed-812 resin in BEEM capsules. ${ }^{12}$ Ultrathin sections were cut and stained with uranyl acetate and lead citrate and examined under JEOL JEM 1010 electron microscope in Electron Microscope Research Laboratory (EMRL), Department of Histology, Faculty of Medicine, Zagazig University. Olympus CX 41 image analyzer software was used for image analysis and morphometric study. The diameter of the whole urinary bladder and the thickness of its muscle layer were measured.

4. Statistical analysis was performed using Statistical Package for the Social Sciences software (SPSS version 22.0) . Data were analyzed using one way ANOVA followed by LSD. $P$ value $\leq 0.05$ was considered significant. Results were expressed as means \pm SD.

\section{RESULTS}

\section{Histopathological Analysis}

Histopathological analysis of the bladder tissue of group (A) showed normal features of epithelium and muscolosa [Figure 1.]. Meanwhile, the bladder tissue of group (B) showed that thickness of transitional epithelium was increased compared to the control group [Figure 2. A\&B]. The smooth muscle cells were hypertrophied with abundant cytoplasm and relatively widely spaced nuclei [Figure 2. C]. Using Mallory's trichrome stain, a marked increase of the collagen fibers in the lamina propria and in between 
the muscle fibers was observed [Figure 2. D]. In group (C), a thinner epithelium was observed compared to the diabetic group. The lamina propria contained collagen fibers and connective tissue cells. Cellular infiltration also appeared in its superficial layer [Figure 3. A\&B]. Smooth muscle bundles were observed in loose connective tissues [Figure 3. C]. By Mallory's Trichrome stain, there were abundant collagen fibers in the lamina propria and in between muscle fibers [Figure 3. D].

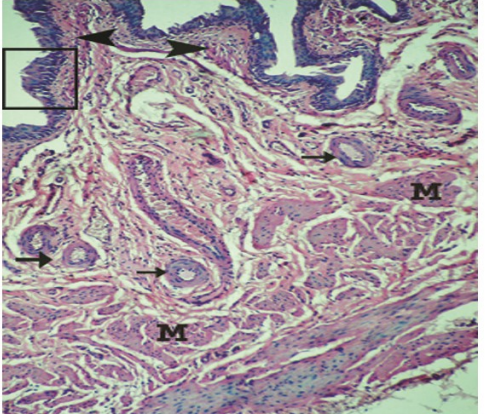

A

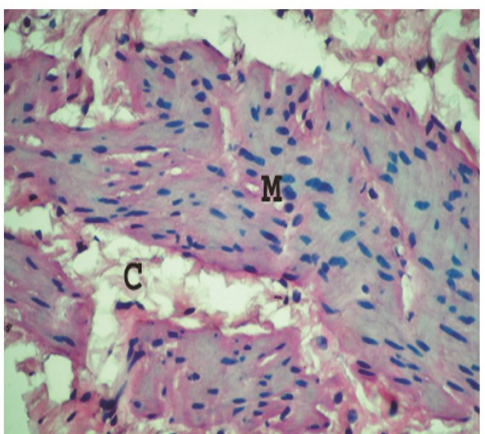

C

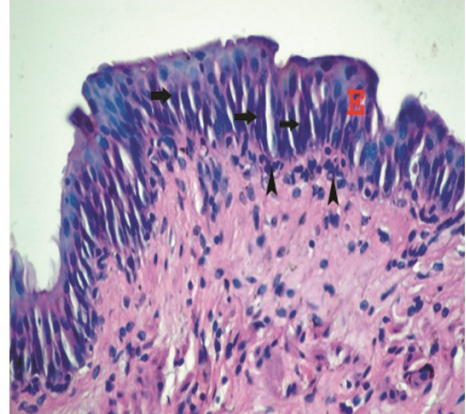

B

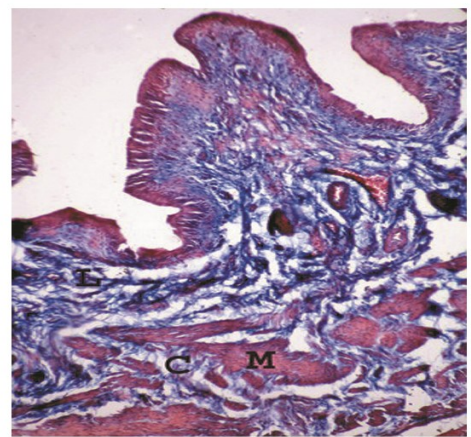

$D$

Figure 1. Histologic features of urinary bladder of the control group. A. Showing normal features of epithelium and muscolosa. Mucosa (I); musculosa (II); serosa (III); transitional epithelium (E); lamina propria (L): musculosa (M); loose connective tissue (C) (H\&E, $\mathrm{x100)}$ ). B. Showing layers of transitional epithelium (E) with prominent acidophilic surface membrane (arrow). The lamina propria is seen in two zones; a dense subepithelial zone (L1) and a deeper loose connective tissue one (L2) (H\&E, x400). C. Showing part of the musculosa with bundles of smooth muscle fibers (M) in different directions. Connective tissue (C) (H\&E, x400). D. Showing collagen fibers (C) in the lamina propria (L) and in between the muscle bundles (M). Blood vessels (arrows) are also noticed in the lamina propria (Masson's Trichrome, x100).
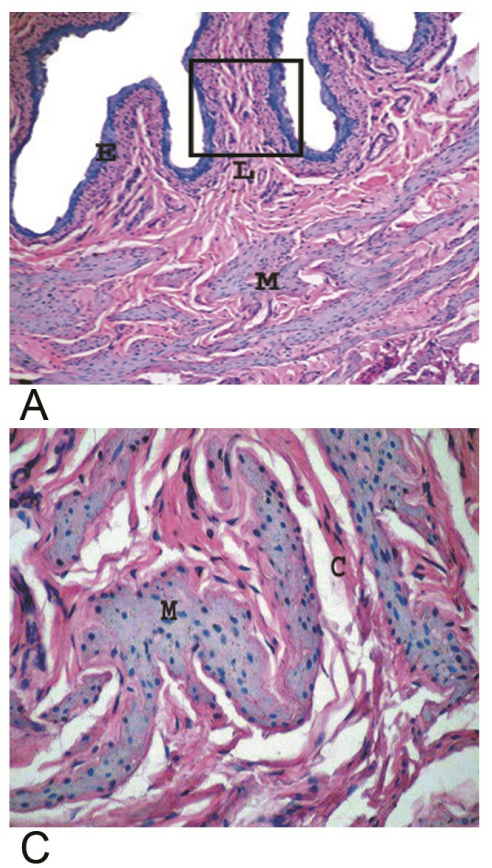
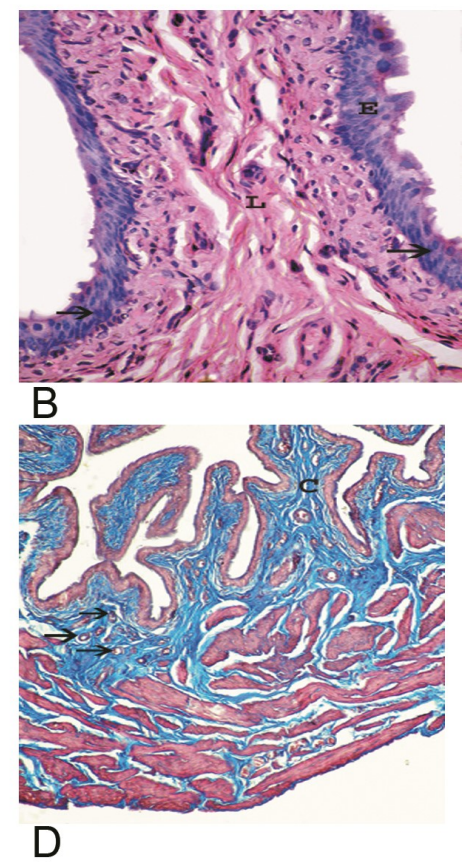

Figure 2. Histologic features of urinary bladder of the diabetic group. A. Showing thick transitional epithelium (E); a thick layer of muscle fibers (M); serosa (S) (H\&E, x100). B. Showing thick layer of transitional epithelium (E) with dark nuclei (arrows); lamina propria (L) consisting of superficial dense and deep loose connective tissue (H\&E, x400). C. Showing smooth muscle fibers (M) with homogeneous cytoplasm and elongated nuclei; collagen fibers (C) (H\&E, x400). D. Showing numerous collagen fibers (C) (Masson's Trichrome, x100). 


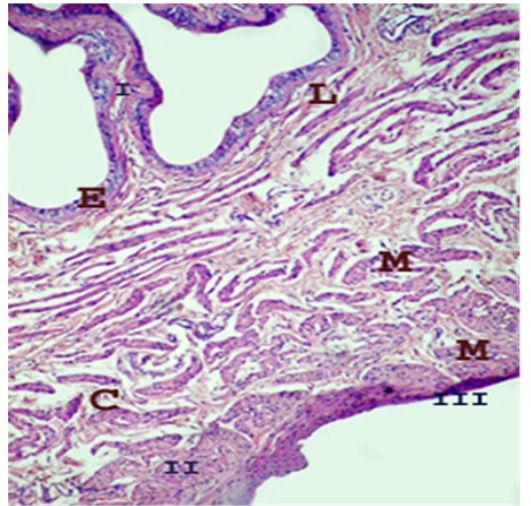

A

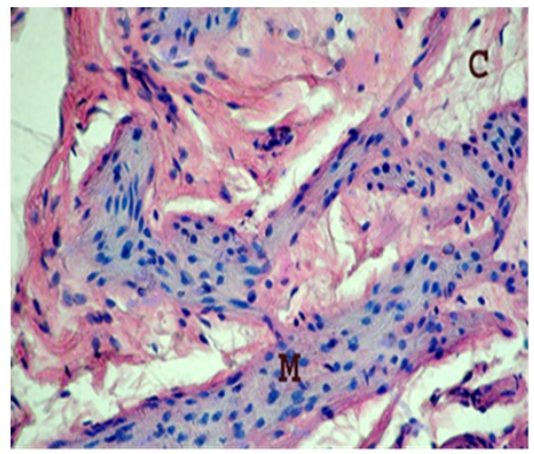

C

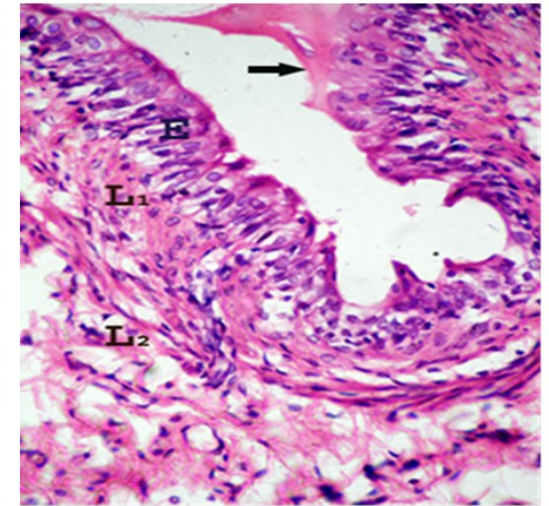

B

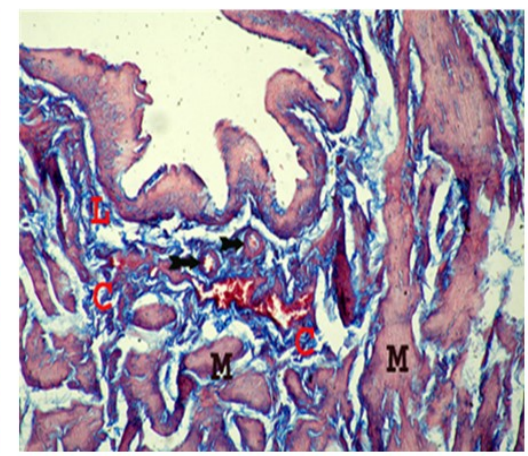

D

Figure 3. Histologic features of urinary bladder of the diabetic group treated with insulin. A. Showing relatively large smooth muscle bundles $(M)$ (H\&E, $x 100)$. B. Showing transitional epithelium (E); darkly stained nuclei (arrows); (H\&E, $x 400)$. C. Showing hypertrophy of smooth muscle fibers $(M)$; interstitial connective tissue (C). (H\&E, x400). D. Showing abundant collagen fibers $(C)$ in the lamina propria and in between the muscle fibers $(M)$; Blood vessels (arrows) (Masson's Trichrome, x100).

\section{Electron Microscopic Analysis}

In Group (A), cells of the transitional epithelium showed extensive cell membrane interdigitations and euchromatic nuclei containing patches of heterochromatin. The luminal border showed angulations and outer thick electron dense line. Moreover, collagen fibrils, fibroblasts, and blood capillaries were observed in the underlying connective tissue [Figure 4. A]. Using higher magnification; irregular intermediate cells, euchromatic nuclei, and minimal intercellular spaces were observed. Cytoplasm showed abundant free ribosomes, little cisternae of the rough endoplasmic reticulum, and few mitochondria [Figure 4. B]. Muscle fibers were outlined by minimal intercellular spaces with central and heterochromatic nuclei. Dense plaques were observed under the sarcolemma [Figure 4. C].

Meanwhile, electron microscopy of the group (B) revealed wide intercellular spaces between the surface epithelium. Cells showed electron-dense cytoplasm and nuclei. The angular luminal border was observed [Figure 5. A\&B]. Mast cells were observed between the superficial cells near the lumen. Their cytoplasm was occupied by granules of different sizes and electron densities [Figure 5. C] Excess collagen fibrils were observed in the lamina propria between the connective tissue cells [Figure 5. D]. Smooth muscle fibers with homogeneous cytoplasm and indented nuclei were noticed [Figure 5. E]. Histological features of group (C) were similar when compared to group (A). Large epithelial cells with euchromatic nuclei containing patches of heterochromatin and extensive interdigitations were observed [Figure 6. A\&B]. Smooth muscle fibers with central and heterochromatic nuclei were observed [Figure 6. C].

\section{Morphometric Analysis}

Morphometric measurements of the bladder diameter of all groups showed an obvious increase in the diameter of bladder in diabetic group compared to control and insulin-treated groups [Table 1]. Moreover, assessment of epithelial and muscle thickness revealed that the urinary bladder of the diabetic group showed a significant $(P$ value $<0.05)$ increase in epithelial thickness [Table 1] and smooth 
muscle layer thickness [Table 1] compared to control group. Meanwhile, results of the control and insulin-treated groups were insignificantly different.

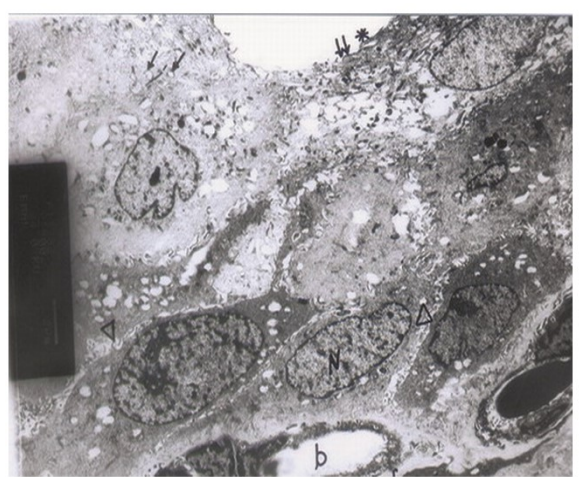

A

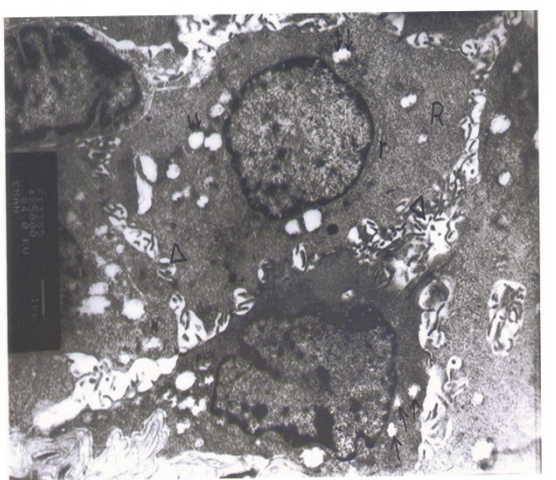

B

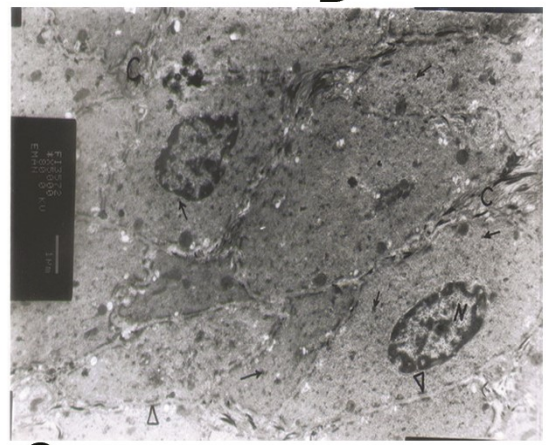

C

Figure 4. Electron microscopic features of urinary bladder of the control group. A. Showing transitional epithelium with lenticular vesicles (arrows); cell membrane interdigitations (head arrow); euchromatic nuclei (N); angulated luminal border (star); thick outer dense line (double arrows); blood capillaries (b); fibroblast (f) (x6000). B. Showing middle and basal layers with cellular interdigitations (head arrows); lenticular vesicles (double arrows); rough endoplasmic reticulum ( $r$ ); ribosomes $(R)$; mitochondria (arrows) ( $x 12000)$. C. Showing smooth muscle fibers with heterochromatic nuclei (N); collagen fibrils (C); dense bodies (arrows). Dens plaques (head arrow) (x12000).

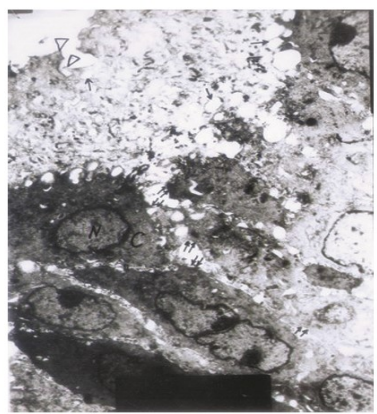

A

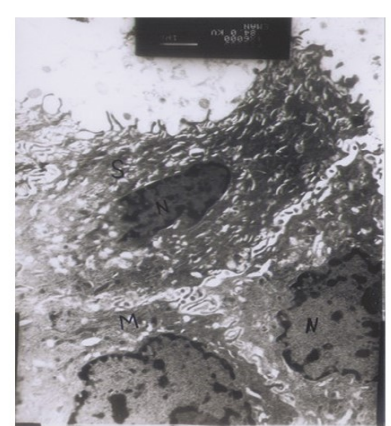

B

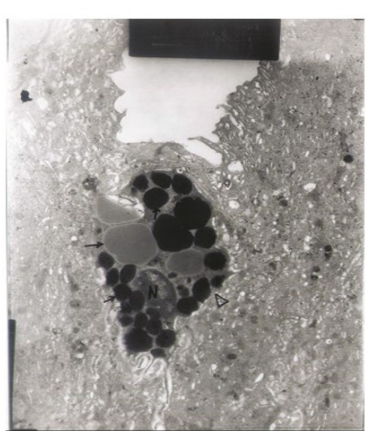

C
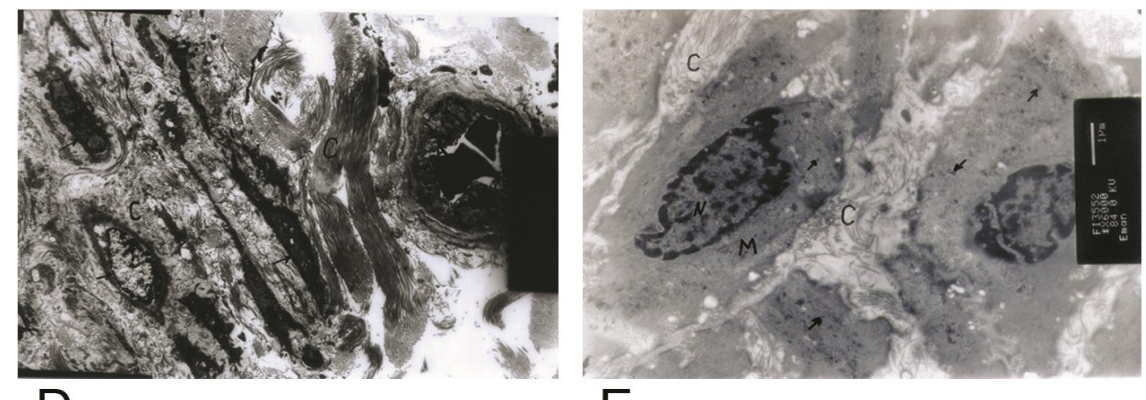

E

Figure 5. Electron microscopic features of urinary bladder of the diabetic group. A. Showing the transitional epithelium with wide intercellular spaces (double arrows); electron-dense cytoplasm (C) and nuclei (N); Large lenticular vesicles (arrows); angulated luminal border (head arrow) (x6000). B. Showing electron-dense cytoplasm in superficial (S) and middle $(M)$ layers of the transitional epithelium; irregular indented electron dense nuclei $(\mathrm{N})$ containing peripheral heterochromatin (x13000). C. Showing intraepithelial mast cell (head arrows); granules of different electron densities (arrows); heterochromatic nucleus $(N)(x 12000)$. D. Showing abundant collagen fibrils $(C)$ between the connective tissue cells (arrows); an arteriole (A) (x6000). E. showing smooth muscle cells (M); few dense bodies (arrows); nuclei (N); thick peripheral heterochromatin; wide intercellular spaces filled with collagen fibrils (C) (x14000). 
Table 1 Diameter, epithelial thickness, and muscle thickness of experimental groups

\begin{tabular}{cccc}
\hline & $\begin{array}{c}\text { Control } \\
\text { group }\end{array}$ & Diabetic group & $\begin{array}{c}\text { Insulin } \\
\text { treated group }\end{array}$ \\
\hline $\begin{array}{c}\text { Diameter } \\
\text { (Pixels) }\end{array}$ & $646.7 \pm$ & ${ }^{*} 1839.98 \pm$ & $938.7 \pm$ \\
$\begin{array}{c}\text { Epithelial } \\
\text { thickness } \\
\text { (pixels) }\end{array}$ & $133.6 \pm$ & $* 331.8 \pm$ & $141.4 \pm$ \\
$\begin{array}{c}\text { Muscle } \\
\text { thickness } \\
\text { (pixels) }\end{array}$ & 11.9 & 27.1 & 16.8 \\
\hline
\end{tabular}

\section{DISCUSSION}

Diabetic cystopathy is a well-known complication of diabetes mellitus which occurs in $25-85 \%$ of cases. ${ }^{13}$ It is manifested by an impaired bladder sensation, an increase in bladder capacity, and a reduction in detrusor muscle contractility. Other signs including detrusor overactivity, urgency, and frequency are further reported. ${ }^{13}$ These complications of diabetes are typically not life-threatening but can be very debilitating as they often progress throughout the patient's life and are associated with significant healthcare economic burden. Despite their common occurrence, the mechanism of diabetes-related urological problems is not well understood. ${ }^{14}$ Previous studies revealed the damaging effects of STZ induced-diabetes on bladder tissue. STZ induced-diabetes contributed to the early development and progression of diabetic renal interstitial fibrosis. ${ }^{8}$ Moreover, early development of thick detrusor muscle in STZ-induced diabetic rats was noticed which was mainly contributed to the markedly altered expression of the gap junction protein $\mathrm{Cx} 43$ in the urinary bladder tissue. ${ }^{9}$

In the present study, the transitional epithelium of the urinary bladder of the control group showed normal structure consisting of many layers arranged in three layers of cells: superficial, intermediate and basal cells. However, the variability observed in the number of layers may be due to the plane of the section or the degree of distension of the bladder. ${ }^{15-16}$

On the other hand, examination of the transitional epithelium of the diabetic group showed an increase in its thickness with widening of the intercellular spaces. Moreover, the nuclei were darkly stained. This was in accordance with Harry et al. ${ }^{17}$ who reported an increase in expression of $B$ actin, a gene product associated with cell structure, in bladders as early as the first week following induction of diabetes which indicated cellular hypertrophy. Furthermore, Pinna et al. ${ }^{18}$ mentioned that there was a progressive increase in the thickness of urothelium of diabetic rats due to the increase in DNA synthesis. On the other hand, we found out that the diabetic group treated with insulin revealed a normal thickness of epithelium which was quite similar to the control group.

Electron microscope examination of the transitional epithelium of the diabetic group revealed widened intercellular spaces with numerous and large lenticular vesicles. Some cells appeared with electron-dense cytoplasm. Similar findings were reported by Rizk et al. ${ }^{5}$ who stated that some of the deeper cells of the urothelium had become electron dense two months after induction of diabetes. It was suggested that these changes were a part of a pre-apoptotic stage. Apoptosis was also reported by Staiger et al. ${ }^{19}$ who reported an increase in the apoptotic cells in endothelial cells and smooth muscle cells of coronary arteries in diabetic rats. Sharma et al. ${ }^{20}$ and Seven et al. ${ }^{21}$ reported a rise in cellular damage in bladders of diabetic rats and it was attributed to the liberation of free radicals. In contrast, Khan et al. ${ }^{22}$ showed an increase in cellular proliferation and a decrease in apoptosis in the urothelial cells of urinary bladders six months after induction of diabetes.

In the diabetic group treated with insulin, we noticed that the cells of the transitional epithelium showed minimal intercellular spaces, prominent euchromatic nuclei, and numerous mitochondria. This may suggest that insulin may prevent or delay the adverse effects of diabetes on the epithelium. Staiger et al. ${ }^{19}$ reported that treatment with insulin prevented cellular apoptosis that occurred in the endothelium of the coronary artery of the diabetics. In the present study, examination of the lamina propria of the control group revealed normal architecture. Eika et al. ${ }^{10}$ reported that the collagen component of the bladder wall changes in amount as well as in quality in the diabetic rats. Bezuijen et al. ${ }^{23}$ attributed the increase in production of collagen to the hyperactive detrusor myocytes and stromal fibroblasts. We noticed that the lamina propria of the diabetic rats treated with insulin showed a slight increase in the collagen fibers compared to the control group. However, Eika et al. ${ }^{10}$ reported that insulin was able to prevent 
the changes in collagen fibers that occurred in the diabetic bladder.

Mast cells were observed in the lamina propria and in between the cells of urothelium in diabetic rats of this study. Kuroki et al. ${ }^{24}$ reported the same findings and it was attributed to the inflammatory conditions which are common with diabetes. Mast cells play an important role in allergic conditions by initiating inflammatory response known as immediate hypersensitivity reaction due to exposure to antigen. The first exposure to antigen leads to sensitization of mast cells by binding of IgE antibodies to the receptors of the plasmalemma of mast cells. On a subsequent exposure to the same antigen, it binds to IgE on mast cell surface leading to release of mediators such as histamine, leukotriene, prostaglandins, and bradykinins. We noticed the presence of inflammatory cells in the lamina propria of the diabetic rats treated with insulin indicating the presence of infection. Edward et al. ${ }^{25}$ reported an increase in the frequency of urinary tract infection in severe cases of diabetic women treated with insulin.

In the present investigation, the diabetic group revealed numerous congested blood vessels in the lamina propria. The process of development of new blood vessels (angiogenesis) is induced by a potent growth factor known as vascular endothelial growth factor which is released into the bloodstream as a result of complications related to diabetes ${ }^{26}$. However, Tamart et al. ${ }^{27}$ mentioned that the rise in the formation of advanced glycation end products (AGEs) was generally regarded as one of the main mechanisms responsible for vascular changes in patients with diabetes.

The smooth muscle fibers of the diabetic group were swollen and hypertrophied. Moreover, the intercellular spaces were wide and filled with collagen fibers. Lincoln et al. ${ }^{28}$ reported hypertrophy of the smooth muscle of the bladder wall in experimental diabetic rats. Ayan et al. ${ }^{13}$ reported that the disturbance of detrusor muscle function was due to the affection of the nerve fibers which were observed to be beaded, vacuolated, and fragmented. Furthermore, Harry et al. ${ }^{17}$ found that the sympathetic and sensory neurons were affected at an early stage in diabetes. It was suggested that diabetes causes a delay in the first sensation to void bladder during its filling and that leads to an increase in bladder capacity which was interpreted as sensory impairment. ${ }^{29}$ This altered urodynamic response with development of poor contractility of the hypertrophied muscle leads to impaired bladder emptying and residual urine. Despite all theories explaining the adverse effects of diabetes on urinary bladder, the exact underlying mechanism of diabetes-induced alteration in detrusor smooth muscle contractility and bladder dysfunction are not yet well understood.

Insulin-treated group showed relatively thick bundles of muscle fibers. Myocytes showed dense bodies in the cytoplasm and dense plaques under the sarcolemma. Al Jaroudi et al. ${ }^{30}$ reported that muscle hypertrophy did not occur in the heart of diabetic rats treated with insulin and concluded that early treatment with insulin prevents many complications. Our results revealed an increase in thickness in the epithelium and muscle layer of the bladder in the diabetic group compared to the control and insulintreated group. A previous study reported thickening of muscolosa layer of the bladder along with the loss of nerve function and anatomical loss of neuromuscular nerve terminals in diabetic rats. ${ }^{3}$

\section{CONCLUSION}

The present study revealed the damaging effect of STZ-induced diabetes on the urinary bladder of rats. Thickening of mucosa and muscolosa, abundant deposition of collagen fibers in the lamina propria, and wide intercellular spaces were observed in the urinary bladder of diabetic animals. However, treatment with insulin was found to be effective in preventing these adverse effects. Thus, early control of diabetes and frequent evaluation of bladder function are recommended for diabetic patients to prevent or at least delay the complications.

\section{CONFLICT OF INTEREST}

No potential conflict of interest relevant to this article was reported.

\section{ACKNOWLEDGMENT}

The research was funded by a research grant from Faculty of Medicine, Cairo University. 


\section{REFERENCES}

1. Arulmozhi D, Veeranjaneyulu A, Bodhankar S. Neonatal streptozotocin-induced rat model of Type 2 diabetes mellitus: A glance. Indian J Pharmacol 2004; 36:217-21.

2. Dobretsov M, Romanovsky D, Stimers JR. Early diabetic neuropathy: triggers and mechanisms. World J Gastroenterol 2007; 13:175-91.

3. Poladia D, Schanbacher B, Wallace L, Bauer J. Innervation and connexin isoform expression during diabetes-related bladder dysfunction: early structural vs. neuronal remodelling. Acta Diabetol 2005; 42:147-52.

4. Castro JC, Camargos AF, Goulart EA, Junqueira HS. Sonographic evaluation of bladder volume in diabetic children and adolescents compared with nondiabetic subjects. J Ultrasound Med 2004; 23:1307-13.

5. Rizk DE, Padmanabhan RK, Tariq S, Shafiullah M, Ahmed I. Ultra-structural morphological abnormalities of the urinary bladder in streptozotocin-induced diabetic female rats. Int Urogynecol J 2006; 17:143-54.

6. Steers WD, Tuttle JB. Mechanisms of disease: the role of nerve growth factor in the pathophysiology of bladder disorders. Nat Clin Pract Urol. Urology 2006; 3:101-10.

7. Poladia DP, Bauer JA. Functional, structural, and neuronal alterations in urinary bladder during diabetes: investigations of a mouse model. Pharmacol 2005; 74:84-94.

8. Li J, Qu X, Bertram JF. EndothelialMyofibroblast Transition Contributes to the Early Development of Diabetic Renal Interstitial Fibrosis in Streptozotocin-Induced Diabetic Mice. Am J Pathol 2009; 175: 1380-8.

9. Suadicani SO, Urban-Maldonado M, Tar MT, Melman A, Spray DC. Effects of ageing and streptozotocin-induced diabetes on connexin43 and $\mathrm{P} 2$ purinoceptor expression in the rat corpora cavernosa and urinary bladder. BJU Int 2009; 103: 1686-93.

10. Eika B, Levin RM, Longhurst PA. Collagen and bladder function in streptozotocin-diabetic rats: effects of insulin and aminoguanidine. J Urol 1992; 148:167-72.

11. Bancroft JD, Gamble M. Theory and practice of histological techniques. London, Edinburgh, New York: Churchill Livingstone/Elsevier; 2002.
12. Glauert AM, Lewis PR. Biological Specimen Preparation for Transmission Electron Microscopy. USA: Princeton University Press; 1998.

13. Ayan S, Kaloglu C, Ucar GGC, Kilicarslan H, Gultekin E. Effect of insulin therapy for diabetic cystopathy: Urodynamic and histological findings in a rabbit model. Scand J Urol Nephrol 1999; 33:392-5.

14. Pitre D, Ma T, Wallace L, Bauer J. Timedependent urinary bladder remodeling in the streptozotocin-induces diabetic rat model. Acta Diabetol 2002; 39:23-7.

15. Jost SP, Gosling JA, Dixon JS. The morphology of normal human bladder urothelium. J Anat 1989; 167:103-15.

16. Komissarchik $Y$, Snigirevskaya $E$, Shakhmatova $E$, Natochin Y. Ultrastructural correlates of the antidiuretic hormone-dependent and antidiuretic hormone-independent increase of osmotic water permeability in the frog urinary bladder epithelium. Cell Tissue Res 1998; 293:517-24.

17. Koo HP, Santarosa RP, Buttyan R, et al. Early molecular changes associated with streptozotocin-induced diabetic bladder hypertrophy in the rat. Urol Res 1993; 21:37581.

18. Pinna C, Zanardo R, Puglisi L. Prostaglandinrelease impairment in the bladder epithelium of streptozotocin-induced diabetic rats. Eur J Pharmacol 2000; 388:267-73.

19. Staiger $K$, Staiger $H$, Schweitzer $M$, et al. Insulin and its analogue glargine do not affect viability and proliferation of human coronary artery endothelial and smooth muscle cells. Diabetologia 2005; 48:1898-905.

20. Sharma A, Kharb S, Chugh S, Kakkar R, Singh $G$. Evaluation of oxidative stress before and after control of glycemia and after vitamin $\mathrm{E}$ supplementation in diabetic patients. Metabolism 2000; 49:160-2.

21. Sevenl A, Gfizell S, Seymenz O, et al. Effects of Vitamin E Supplementation on Oxidative Stress in Streptozotocin Induced Diabetic Rats: Investigation of. Yonsei Med J 2004; 45:703-10.

22. Khan MA, Thompson CS, Calvert RC, Mikhailidis DP, Morgan RJ. Decreased urinary bladder apoptosis in a rabbit model of diabetes mellitus. Urolithiasis 2002;30: 79.

23. Bezuijen MW, Levendusky MC, Longhurst PA. Functional response of bladder strips from 
streplozotocon diabetic rats depends on bladder mass. J Urol 2003; 169:2397-401.

24. Kuroki T, Isshiki K, King GL. Oxidative stress: the lead or supporting actor in the pathogenesis of diabetic complications. J Am Soc Nephrol 2003; 14:S216-S20.

25. Boyko EJ, Fihn SD, Scholes D, Abraham L, Monsey B. Risk of urinary tract infection and asymptomatic bacteriuria among diabetic and nondiabetic postmenopausal women. Am J Epidemiol 2005; 161:557-64.

26. Vranes D, Cooper ME, Dilley RJ. Cellular mechanisms of diabetic vascular hypertrophy. Microvasc Res 1999; 57:8-18.

27. Tamarat R, Silvestre J-S, Huijberts $M$, et al. Blockade of advanced glycation end-product formation restores ischemia-induced angiogenesis in diabetic mice. Proc Natl Acad Sci 2003; 100: 8555-60.

28. Lincoln J, Haven A, Sawyer M, Burnstock G. The Smooth Muscle of Rat Bladder in the Early Stages of Streptozotocin-induced Diabetes. BJU Int 1984; 56: 24-30.

29. Chang S, Hypolite JA, Disanto ME, et al. Increased basal phosphorylation of detrusor smooth muscle myosin in alloxan-induced diabetic rabbit is mediated by upregulation of Rho-kinase $B$ and CPI-17. Am J Physiol Renal Physiol 2006; 290: F650-F6.

30. Al Jaroudi WA, Nuwayri-Salti N, Usta JA, et al. Effect of insulin and angiotensin II receptor subtype-1 antagonist on myocardial remodelling in rats with insulin-dependent diabetes mellitus. J Hypertens 2005; 23: 381-92. 
